\title{
Impact of Nicotine Consumption on Hyper Acidic Patients Taking PPI: An In-Vitro and Computational Analysis
}

\author{
Sabia Nawsheen1, Md. Abu Sufian1,2, Kanij Nahar Deepa1,3* \\ ${ }^{1}$ Department of Pharmacy, University of Asia Pacific, Dhaka, Bangladesh \\ ${ }^{2}$ Division of Computer Aided Drug Design, Red-Green Research Center, BICCB, Dhaka, Bangladesh \\ ${ }^{3}$ Department of Pharmaceutical Chemistry, Faculty of Pharmacy, University of Dhaka, Dhaka, Bangladesh \\ Email: *kanijdeepa@uap-bd.edu
}

How to cite this paper: Nawsheen, S., Sufian, Md.A. and Deepa, K.N. (2019) Impact of Nicotine Consumption on Hyper Acidic Patients Taking PPI: An In-Vitro and Computational Analysis. Health, 11, 129-141.

https://doi.org/10.4236/health.2019.112012

Received: December 23, 2018

Accepted: February 10, 2019

Published: February 13, 2019

Copyright $\odot 2019$ by author(s) and Scientific Research Publishing Inc. This work is licensed under the Creative Commons Attribution International License (CC BY 4.0).

http://creativecommons.org/licenses/by/4.0/

\begin{abstract}
The study aims to investigate the protein binding kinetics of nicotine and a PPI (pantoprazole) with Bovine Serum Albumin (BSA) through UV spectroscopy and computational modeling. Data was obtained by using nicotine and pantoprazole and warfarin and diazepam as the two site specific probes on Bovine serum albumin (BSA). In-vitro and in-silico modeling was carried out in creating an environment that simulates the body environment. Cellulose membrane tubes were cut into $9 \mathrm{~cm}$ and tied tightly not to let any mixtures leak out. To determine number of binding sites, association constants by using Scatchard plot, predominant binding site of each drug and rise in \% of free fraction of one by the other were analyzed using equilibrium dialysis method. Molecular docking further verifies the observations. In Scatchard plot analysis, for nicotine, $\mathrm{n}_{1}, \mathrm{n}_{2}, \mathrm{k}_{1}$ and $\mathrm{k}_{2}=2.2,7.6,0.18 \mu \mathrm{M}^{-1}$ and $0.02 \mu \mathrm{M}^{-1}$ and for pantoprazole, $\mathrm{n}_{1}, \mathrm{n}_{2}, \mathrm{k}_{1}$ and $\mathrm{k}_{2}=0.42,1.2,0.40 \mu \mathrm{M}^{-1}$ and $0.03 \mu \mathrm{M}^{-1}$. Nicotine binds more to diazepam site (site-II) and pantoprazole mainly to warfarin site (site-I). In molecular docking, the binding affinity of nicotine being $-5.7 \mathrm{kcal} / \mathrm{mole}$ demonstrates higher affinity for site-II than that of pantoprazole whose binding affinity is $-8.0 \mathrm{kcal} / \mathrm{mole}$. In absence and presence of warfarin, the free fraction of pantoprazole bound to BSA (1:1) was increased from $37.79 \%$ to $82.44 \%$ and $51.78 \%$ to $98.80 \%$ respectively by nicotine. On the other hand, free fraction of nicotine was raised by pantoprazole from $12.89 \%$ to $75.70 \%$ and $50.08 \%$ to $99.66 \%$ in the absence and presence of diazepam. Both the results of spectroscopic and computational molecular docking suggest that administering pantoprazole with nicotine might increase the $\%$ free fraction of pantoprazole more. Thus, nicotine consumption can be beneficial for smoker people taking PPI like pantoprazole.
\end{abstract}




\section{Keywords}

Nicotine, Pantoprazole, BSA, Protein Binding, Equilibrium Dialysis, Molecular Docking

\section{Introduction}

Nicotine, is a highly addictive alkaloid [1] found in the leaves of Nicotianarustica, Nicotiana tabacum, Duboisia hopwoodii and Asclepias syriaca and has been used as an insecticide in the past [2]. An average cigarette yields about $2 \mathrm{mg}$ of absorbed nicotine, the substance acts as a stimulant in mammals, while high amounts (50 - $100 \mathrm{mg}$ ) can be harmful [3]. Nicotine's addictive nature includes psychoactive effects, gastric effects, drug-reinforced behavior, compulsive use, and relapse after abstinence, physical dependence and tolerance [4] which is also linked to possible birth defects [5] [6]. Pantoprazole, on the other hand, is a proton pump inhibitor that inhibits gastric acid secretion and treats gastric ulcers by irreversibly blocking the proton pump [7]. There is a lower esophageal sphincter function of chronic smokers compared with non-smokers. Cigarette smoking probably exacerbates reflux disease by directly provoking acid reflux and perhaps by a long lasting reduction of lower esophageal sphincter pressure [8]. So, smoking increases the risk of heartburn and GERD and also peptic ulcers for which smokers take omeprazole or pantoprazole for better functioning of the body.

Serum albumin is the primary extracellular protein of the circulatory system contributing to about $60 \%$ mass of plasma proteins [9]. It serves as a transport carrier for many drugs and plays a dominant role on their bioavailability [10]. The interaction of any drug with serum albumin is highly characterized by the properties of the drug, namely, its concentration, physicochemical properties, and also by number of vacant binding sites on the protein and $\mathrm{pH}$ of the medium [11]. Consequently, the synergy between the drug molecule and albumin exerts considerable effects on the pharmacodynamics and pharmacokinetics, i.e., distribution, metabolism and excretion of the drug when stable or reversible drug-protein complexes are formed [12] [13]. Such effects are mainly altered when two or more drugs are administered simultaneously as one drug displaces the other from its binding site, increases its free fraction and hence causes a noticeable rise in activity [14] [15]. Thus, if nicotine and pantoprazole are co-administered, the pharmacokinetics of one or both might alter due to their simultaneous presence in the albumin, even though, the drugs bind on the different binding sites. For their simultaneous administration, we need to know the binding kinetics of these two drugs with serum albumin, i.e., number of binding sites for each drug, their predominant binding sites, displacement of one drug from the binding pocket of albumin by the other and rise of $\%$ free fraction of one drug by the other. 
Our study attempts to examine the interaction of nicotine and pantoprazole with bovine serum albumin (BSA) and the effect on \% free fraction of one drug bound to BSA by the other by spectroscopic and computational tools. We chose BSA as the model protein since it bears approximately $76 \%$ of similarity with human serum albumin (HSA) [16]. Moreover, BSA has $88 \%$ of similarity in amino acid sequence with HSA and hence 3D structure of BSA is believed to be a close match to that of HSA. Furthermore, BSA was preferred over HSA owing to its wider availability at highly pure form, lower cost, high stability, and medical value. Warfarin and diazepam are used in our study as those are Site I and Site II specific probes respectively on BSA protein [10] [17] [18].

\section{Methods}

\subsection{Study Design}

The kinetics of nicotine and pantoprazole interactions with BSA were studied separately in vitro by equilibrium dialysis and UV spectroscopy [18]. The 3D chemical structures of nicotine, pantoprazole, warfarin sodium and diazepam were retrieved from PubChem Open Chemistry Database [19]. The 3D crystal structure of BSA with selected PDB ID (4JK4) was obtained from Protein Data Bank (PDB) database (https://www.rcsb.org/structure/4jk4).

\subsection{Sample}

Pantoprazole and warfarin sodium were kind gifts from Incepta Pharmaceuticals Ltd, Bangladesh. Diazepam and nicotine were received from Square Pharmaceuticals Ltd., Bangladesh and Vapes needs alltime, Dhaka, Bangladesh respectively. BSA (product number: A 5611) was purchased from Sigma-Aldrich. Spectra/PorCellulose membrane (MWCO $6-8 \mathrm{KD}$, Width $23 \mathrm{~mm}$ ) was purchased from local supplier. BSA stock solution was prepared in phosphate buffer solution (PBS) ( $\mathrm{pH} 7.4)$ at $20 \mu \mathrm{M}$ concentration. Pantoprazole, nicotine, warfarin sodium and diazepam stock solutions were prepared in PBS (pH 7.4) at 100 $\mu \mathrm{M}$ concentration. Working concentrations were prepared by diluting in PBS ( $\mathrm{pH}$ 7.4). Chemical structures of nicotine, pantoprazole, diazepam and warfarin are shown in Figure 1.

\subsection{Measures}

\subsubsection{Estimation of Association Constants and Number of Binding Sites} 10 nicotine-BSA solutions $(0 \mu \mathrm{M}, 25 \mu \mathrm{M}, 30 \mu \mathrm{M}, 35 \mu \mathrm{M}, 40 \mu \mathrm{M}, 45 \mu \mathrm{M}, 50 \mu \mathrm{M}$, $55 \mu \mathrm{M}, 60 \mu \mathrm{M}$ and $65 \mu \mathrm{M} ; 5 \mathrm{ml}$ each) and 10 pantoprazole-BSA solutions (0 $\mu \mathrm{M}, 10 \mu \mathrm{M}, 20 \mu \mathrm{M}, 30 \mu \mathrm{M}, 40 \mu \mathrm{M}, 50 \mu \mathrm{M}, 60 \mu \mathrm{M}, 70 \mu \mathrm{M}, 80 \mu \mathrm{M}$ and $90 \mu \mathrm{M} ; 5$ $\mathrm{ml}$ each) were prepared by taking calculated amounts of nicotine $(1000 \mu \mathrm{M})$, pantoprazole $(100 \mu \mathrm{M})$ and BSA $(20 \mu \mathrm{M})$ solutions. The solutions were then properly mixed and allowed to stand for 30 minutes in order to ensure maximum binding of the drugs with BSA. From each solution, $3.5 \mathrm{~mL}$ was drawn out and filled into previously prepared semipermeable membrane tubes (SS 1). The 
<smiles>CN1CCC[C@H]1c1cccnc1</smiles>

(a)

CI<smiles>Cc1ccc2c(c1)C(c1ccccc1)=NCC(=O)N2C</smiles>

(c)<smiles>COc1ccnc(CSc2nc3cc(OC(F)F)ccc3[nH]2)c1OC</smiles>

(b)<smiles>CC(=O)C(O)C(c1ccccc1)c1c(O)c2ccccc2oc1=O</smiles>

(d)

Figure 1. Chemical structure of (a) nicotine, (b) pantoprazole, (c) diazepam and (d) warfarin.

membrane tubes containing drug-protein mixture were then immersed in separate conical flasks containing $20 \mathrm{ml}$ PBS ( $\mathrm{pH}$ 7.4) and kept in a metabolic shaker (Zhicheng, China) for dialysis for 12 hours $\left(37^{\circ} \mathrm{C}, 40 \mathrm{rpm}\right)$.

\subsubsection{Determination of Predominant Binding Site}

The predominant binding site of nicotine and pantoprazole on BSA was identified in vitro by equilibrium dialysis and UV spectroscopy using warfarin sodium as site-I and diazepam as site-II specific probes. During all the experiments, BSA and the probes (warfarin sodium/diazepam) were taken at a ratio of 1:1 $(20 \mu \mathrm{M}$ : $20 \mu \mathrm{M}$ ) and nicotine or pantoprazole were mixed at an increasing concentration $(0$ to $80 \mu \mathrm{M})$ to get the final ratio of BSA: probe: test drugs of 1:1:0, 1:1:1.5, 1:1:2, 1:1:2.5, 1:1:3, 1:1:3.5, and 1:1:4. All the solutions were allowed for equilibrium dialysis as mentioned earlier. On completion of dialysis, samples were collected from each solution and the free fraction of warfarin sodium and diazepam were measured at 305 and $238 \mathrm{~nm}$.

\subsubsection{Drug Displacement Study}

The effect of pantoprazole on nicotine binding to BSA and vice-versa was evaluated in absence and presence of diazepam and warfarin. At first, keeping the concentrations for nicotine and BSA constant $(20 \mu \mathrm{M}: 20 \mu \mathrm{M})$, pantoprazole was added in increasing concentration $(0$ to $100 \mu \mathrm{M})$ to make final ratio of BSA: nicotine: pantoprazole in each experiment as 1:1:0, 1:1:1, 1:1:2, 1:1:3, 1:1:4 and 1:1:5. While in presence of diazepam, the final ratio of BSA: diazepam: nicotine: pantoprazole were 1:2:1:0, 1:2:1:1, 1:2:1:2, 1:2:1:3, 1:2:1:4 and 1:2:1:5. And for both the cases, dialysis was carried out and absorbance of free nicotine was measured at $260 \mathrm{~nm}$ and amount calculated. Secondly, the estimation for the effect of nicotine on pantoprazole, when bound to BSA was done in a same manner. Here, pantoprazole was kept constant and so the final ratio of BSA:warfarin: pantoprazole:nicotine were 1:2:1:0, 1:2:1:1, 1:2:1:2, 1:2:1:3, 1:2:1:4 and 1:2:1:5. 
And again for both the cases absorbance of free pantoprazole was measured at $220.5 \mathrm{~nm}$ and amount calculated.

\subsubsection{Quantum Chemical Calculations}

This utilized density functional theory (DFT) employing Becke's (B3) exchange functional combining Lee, Yang, and Parr's (LYP) correlation functional [20] [21] and 6-311G $+(\mathrm{d}, \mathrm{p})$ basis set [22]. Molecular orbital calculations were performed using the same level of theory. Hardness $(\eta)$ and softness $(S)$ of the test compounds were calculated from the energies of frontier highest occupied molecular orbitals (HOMOs) and lowest unoccupied molecular orbitals (LUMOs). It considered Parr and Pearson interpretation [23] [24] of harness in DFT and Koopmans theorem [25] on the correlation of ionization potential (I) and electron affinities (E) with HOMO and LUMO energy. The following equations of hardness $(\eta)$ and softness $(S)$ were used.

$$
\begin{gathered}
\eta=(\varepsilon \mathrm{LUMO}-\varepsilon \mathrm{HOMO}) / 2 \\
S=1 / \eta
\end{gathered}
$$

\subsubsection{Molecular Docking}

Docking was performed by Auto Dock Vina software (version 1.1.2) [26]. To dock the compounds against BSA, the center grid box was positioned at the center of the protein structure and was expanded in $\mathrm{x}, \mathrm{y}$ and $\mathrm{z}$ directions until the grid box fully covered the protein structure. During docking the protein structure was kept rigid but torsional rotation was allowed for all rotatable bonds of optimized drug structures to perform flexible docking. The binding affinities of each complex were recorded from vina output. The docked pose of lowest binding free energy conformer for all ligand-BSA complexes were then investigated using Accelrys Discovery Studio 4.1 [27]. The information of non-bonding interactions of the complexes in terms of binding pocket, interface amino acids, binding residues, bond type and bond length were collected. Finally a non-bonding interaction map for each complex was generated to visualize the ligand-BSA interactions.

\subsection{Analysis}

The values of association constants and number of binding sites were estimated by Scatchard plot analysis [10]. For determining of binding sites, subsequent analyses were performed using standard curves (SS 2). In addition to all the above mentioned analysis with the finding of the effect of one drug with another, the unbound fraction in all the cases, from each of the flasks were collected and measured by a UV spectrophotometer (UV 1800, Shimadzu, Japan).

Gaussian 09 program package [28] was used for ligand optimization and molecular orbital calculation. Prior to docking, PyMol (version 1.3) software package program was used to erase all the hetero atoms and water molecules [29] and Swiss-Pdb Viewer software packages (version 4.1.0) helped in energy minimization [30]. 


\section{Results}

\subsection{Association Constants and Binding Sites}

The curvilinear Scatchard graphs of nicotine-BSA and pantoprazole-BSA bindings are presented in Figure 2. Drug-protein binding data reported herein that demonstrates, for each drug there are two different binding sites $\left(\mathrm{n}_{1}\right.$ and $\left.\mathrm{n}_{2}\right)$ and each site is associated with its own independent association constant $\left(k_{1}\right.$ and $\left.k_{2}\right)$. At its low $\left(\mathrm{n}_{1}=2.2\right)$ and high $\left(\mathrm{n}_{2}=7.6\right)$ capacity binding sites, the high and low affinity association constants of nicotine are found to be $0.18 \mu \mathrm{M}^{1}$ and $0.02 \mu \mathrm{M}^{-1}$, respectively. On the other hand, $\mathrm{n}_{1}, \mathrm{n}_{2}, \mathrm{k}_{1}$ and $\mathrm{k}_{2}$ for pantoprazole are $0.42,1.2$, $0.40 \mu \mathrm{M}^{-1}$ and $0.03 \mu \mathrm{M}^{-1}$, respectively. Figure 3 depicts the $\%$ free fraction of the probe $(\mathrm{Pf})$ against the molar ratio of drug and BSA. As observed in case of nicotine that it displaces warfarin sodium $(27.65 \%$ to $64.65 \%)$ way less than that of diazepam (10.5\% to $99.94 \%$ ) from the BSA binding pocket as the molar ratio of nicotine and BSA increases from 0 to 4 . However, pantoprazole raises the \% free fraction of warfarin sodium (10.65\% to $99.15 \%)$ more than that of diazepam (31.96\% to $60.66 \%)$ with the same amount of increase in molar ratio of pantoprazole and BSA as that of nicotine and BSA.

\subsection{Drug Displacement Study}

Figure 4 illustrates the effect of one drug binding with the other to BSA in presence and absence of warfarin sodium and diazepam as site-I and site-II specific probe. The experiments show that \% free fraction of pantoprazole bound to BSA is increased from $37.79 \%$ to $82.44 \%$ and $51.78 \%$ to $98.80 \%$ as the molar ratio of nicotine and BSA goes up from 0 to 5 in absence and presence of warfarin sodium, respectively. On the other hand, rise in \% nicotine free fraction is $12.89 \%$ to $75.70 \%$ and $50.08 \%$ to $99.66 \%$ in absence and presence of diazepam and the same amount of lifting is seen in molar ratio of pantoprazole and BSA, respectively.

\subsection{Quantum Chemical Calculations and Molecular Docking}

Table 1 shows the dipole moment (Debye), HOMO and LUMO energy (eV), LUMO-HOMO energy gap $(\mathrm{eV})$, hardness and softness of the optimized ligands. The calculated dipole moment, and softness are found to be lower for nicotine (3.27 D, and 0.024) than that of pantoprazole (4.29 D and 0.033). On other hand, LUMO-HOMO energy gap and hardness are higher for nicotine $(81.98 \mathrm{eV}$ and 40.99) than that of pantoprazole (59.22 eV and 29.61). The result of molecular docking of nicotine and pantoprazole with BSA is presented in Table 2 in terms of average binding affinity $(\mathrm{kcal} / \mathrm{mole}) \pm$ standard deviation, interaction type, bond distance and interacting residues. Figure 5 and Figure 6 portrays the docked pose of nicotine-BSA and pantoprazole-BSA complexes. The binding affinity of nicotine $(-5.7 \mathrm{kcal} / \mathrm{mol})$ is observed to be quite lesser than that of pantoprazole $(-8.3 \mathrm{kcal} / \mathrm{mol})$ when docked against BSA crystal structure (PDB ID: 4JK4). 


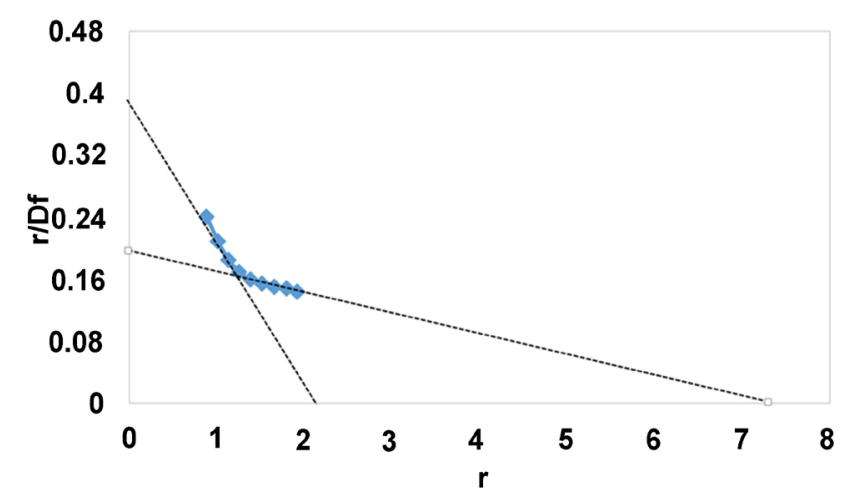

(a)

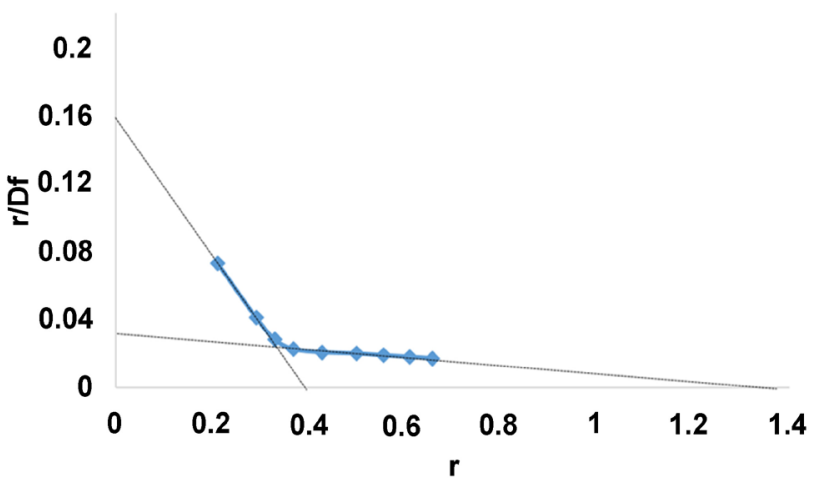

(b)

Figure 2. Scatchard plot of (a) nicotine and (b) pantoprazole bound to bovine serum albumin at $\mathrm{p}^{\mathrm{H}} 7.4^{\circ} \mathrm{C}$ and $37^{\circ} \mathrm{C} . \mathrm{r}=[\mathrm{bound}$ drug] $/[\mathrm{BSA}],\left[\mathrm{D}_{\mathrm{f}}\right]=$ free drug conc., $[\mathrm{BSA}]=20 \mu \mathrm{M}$, [nicotine] $=25-65 \mu \mathrm{M}$, [pantoprazole] $=10-90 \mu \mathrm{M},(\mathrm{a}) \mathrm{n}_{1}, \mathrm{n}_{2}, \mathrm{k}_{1}$ and $\mathrm{k}_{2}=$ $0.87,1.5,0.69 \mu \mathrm{M}^{-1}$ and $0.133 \mu \mathrm{M}^{-1}$, respectively, (b) $\mathrm{n}_{1}, \mathrm{n}_{2}, \mathrm{k}_{1}$ and $\mathrm{k}_{2}=0.42,1.2,0.40 \mu \mathrm{M}^{-1}$ and $0.03 \mu \mathrm{M}^{-1}$, respectively.

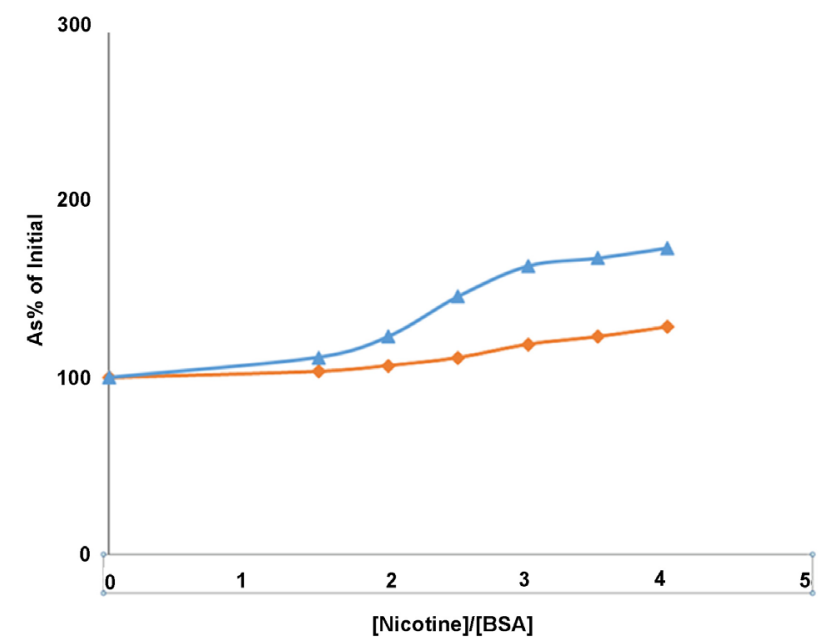

(a)

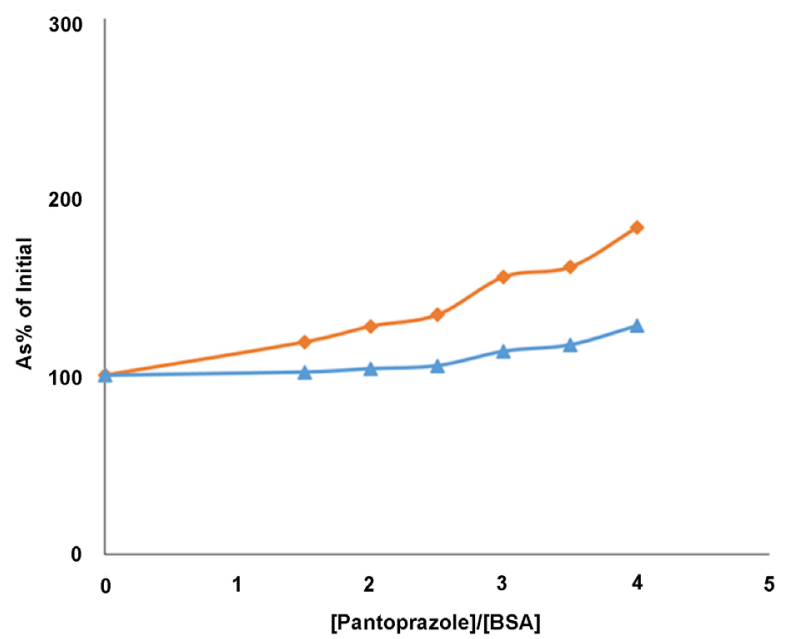

(b)

Figure 3. (a) Free fraction of Warfarin $(>)$ and Diazepam $(\Delta)$ to BSA (1:1) upon the addition of Nicotine and (b) Free fraction of Warfarin ( ) and Diazepam (A) to BSA (1:1) upon the addition of Pantoprazole at $\mathrm{pH} 7.4$ and $37^{\circ} \mathrm{C}$. Concentration of BSA for both the curves, Diazepam and Warfarin was $20 \mu \mathrm{M}$. Nicotine and Pantoprazole concentrations were taken $0-80 \mu \mathrm{M}$.

\section{Discussion}

In the existing study, the potential of co-administration of nicotine and pantoprazole is explored in terms of protein binding kinetics taking bovine serum albumin as the model protein. For each drug, number of binding sites, independent association constants associated with each site, predominant binding site, the increase of $\%$ free fraction of one drug bound to bovine serum albumin by the other are determined by equilibrium dialysis and UV spectroscopic tools. The findings are further verified by molecular docking studies. The number of binding sites of each drug on BSA is determined by Scatchard plot analysis. The curvilinear Scatchard graphs of nicotine-BSA and pantoprazole-BSA binding (Figure 2) indicates that for both of them there are two different binding sites and also 


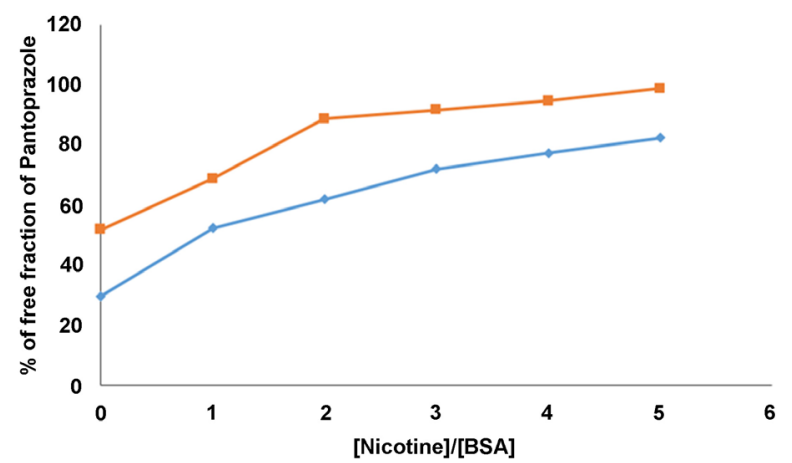

(a)

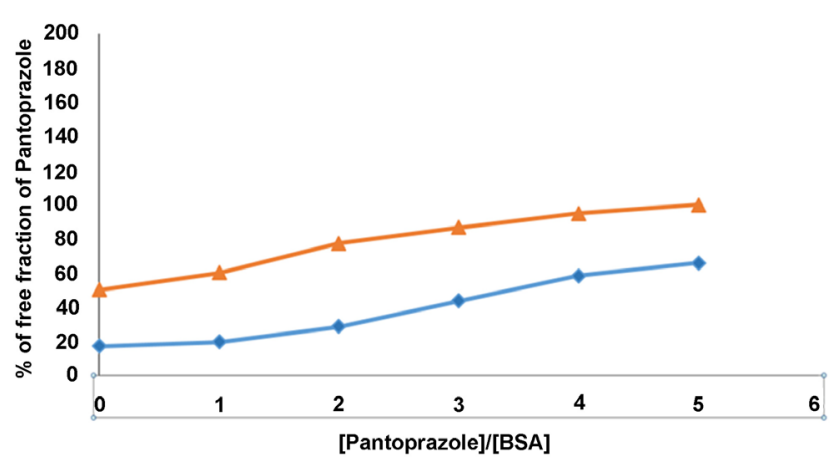

(b)

Figure 4. (a) In presence $(\square)$ and absence $(>)$ of Warfarin, free fraction of Pantoprazole bound to BSA (1:1) upon the addition of Nicotine and $(\mathrm{b})$ in the absence $(>)$ and presence $(\mathbf{A})$ of Diazepam as site II specific probe, free fraction of Nicotine bound to BSA (1: 1) upon the addition of Pantoprazole both at $\mathrm{pH} 7.4$ and $37^{\circ} \mathrm{C},[\mathrm{BSA}]=[$ diazepam $]=[$ warfarin sodium] $=20 \mu \mathrm{M},(\mathrm{a})$ [nicotine] $=0,20,40,80,160 \mu \mathrm{M}$, (b) [pantoprazole] $=0,20,40,80,160 \mu \mathrm{M}$.

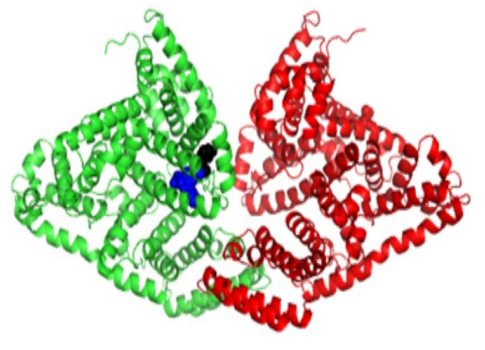

(a)

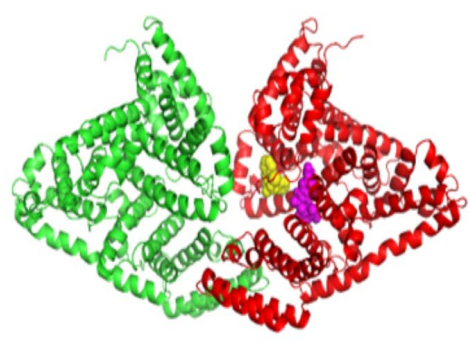

(b)

Figure 5. Nicotine (black), diazepam (blue) bound to chain (b) (green) and pantoprazole (purple), warfarin (yellow) bound to chain (a) (red) of BSA (PDB ID: 4JK4).

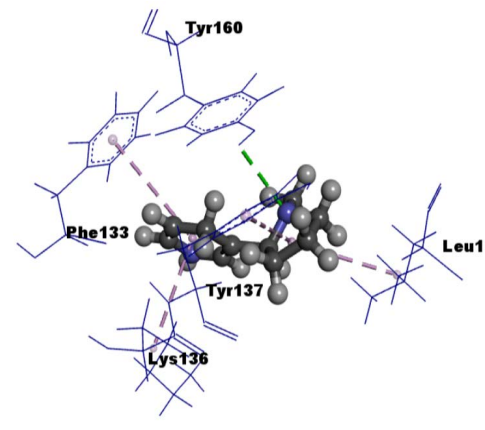

(a)

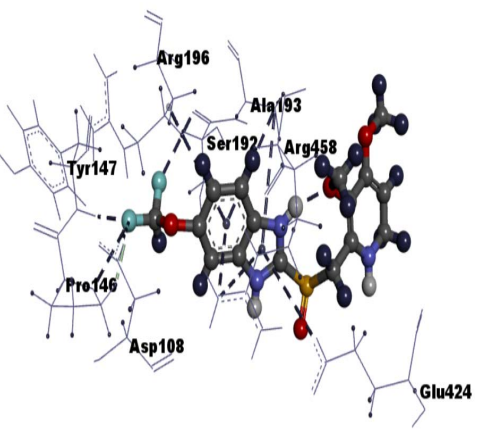

(b)

Figure 6. Nonbonding interaction maps of (a) nicotine and (b) pantoprazole bound to BSA (PDB ID: 4JK4).

two independent association constants associated with each of the site. The association constants of nicotine $\left(0.18 \mu \mathrm{M}^{-1}\right.$ and $\left.0.02 \mu \mathrm{M}^{-1}\right)$ are smaller in magnitude than that of pantoprazole $\left(0.40 \mu \mathrm{M}^{-1}\right.$ and $\left.0.03 \mu \mathrm{M}^{-1}\right)$ is an indication to a higher affinity of pantoprazole than that of nicotine for BSA. Moreover, one association 
Table 1. Dipole moment (Debye), HOMO and LUMO energy (eV), LUMO-HOMO energy gap $(\mathrm{eV})$, hardness and softness of the optimized ligands.

\begin{tabular}{ccccccc}
\hline Ligand & $\begin{array}{c}\mu \\
\text { (Dipole } \\
\text { moment) }\end{array}$ & $\begin{array}{c}\text { HOMO } \\
\text { energy } \\
(\mathrm{eV})\end{array}$ & $\begin{array}{c}\text { LUMO } \\
\text { energy } \\
(\mathrm{eV})\end{array}$ & $\begin{array}{c}\text { LUMO-H } \\
\text { OMO gap }\end{array}$ & $\eta$ (Hardness) & $S$ (Softness) \\
\hline Nicotine & 3.27 & -334.990 & -253.010 & 81.98 & 40.99 & 0.024 \\
Pantoprazole & 4.29 & -306.933 & -247.713 & 59.22 & 29.61 & 0.033 \\
\hline
\end{tabular}

Table 2. Nonbonding interactions of glimepiride and pantoprazole with BSA crystal structure (PDB ID: 4JK4).

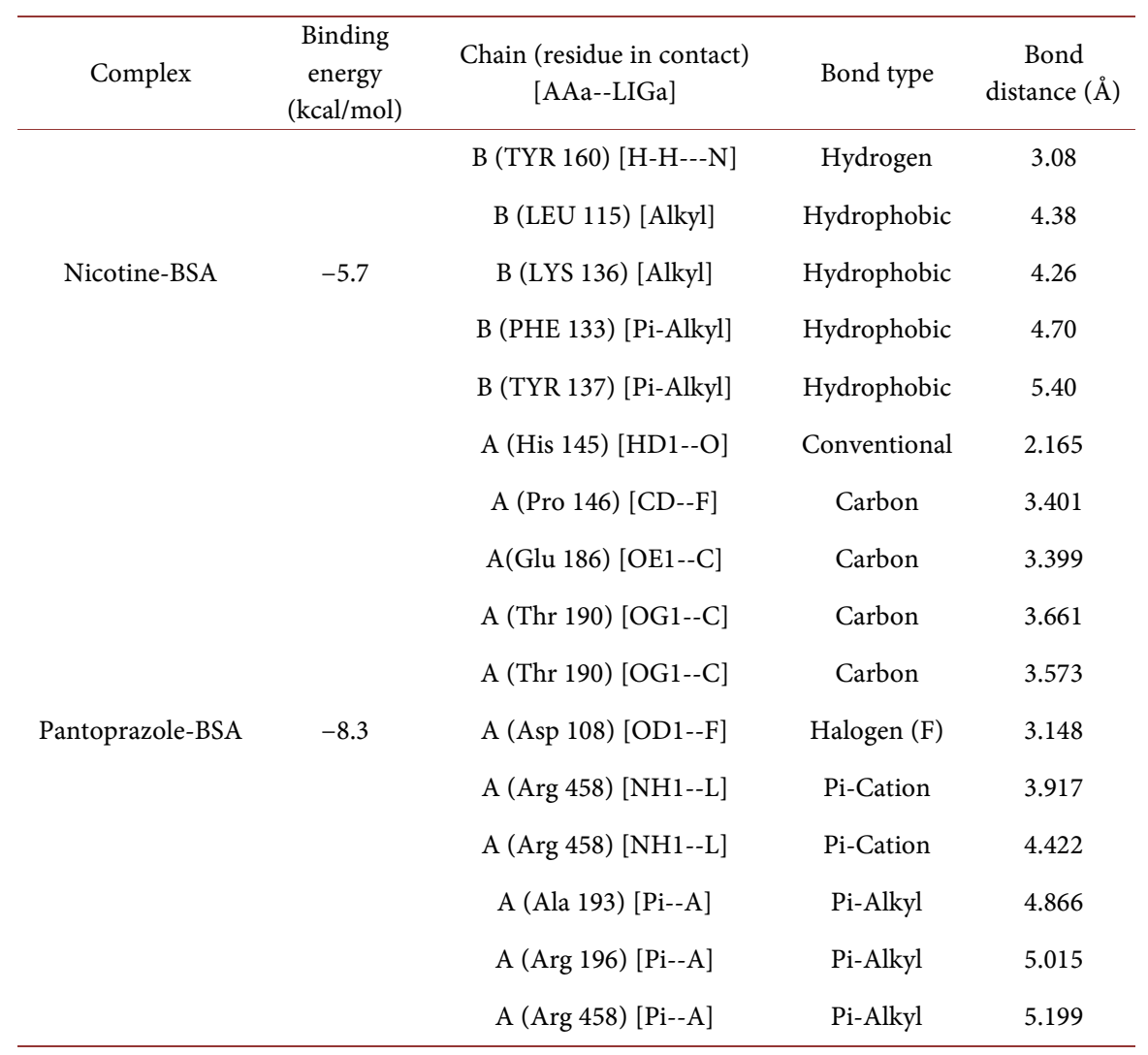

constant being greater than the other testifies that each of the drug predominantly binds to one site of BSA. The predominant binding sites of the test drugs are identified by the degree of displacement they confer to either of the two site specific probes (warfarin sodium as site-I and diazepam as site-II specific probes) of BSA. Here, pantoprazole increases the \% free fraction of warfarin sodium more than diazepam and nicotine increases the $\%$ free fraction of diazepam more than that of warfarin sodium attesting to the fact that one primarily binds to site-I and the other one to site-II ligands of BSA. Besides, the observations in molecular docking are in close harmony with the finding of computational modelling. Pantoprazole and nicotine primarily docks with warfarin sodium and diazepam site of BSA respectively when the grid box size is maximized to cover the entire protein structure (Figure 5). Moreover, average binding af- 
finity of nicotine-BSA complex $(-5.7 \mathrm{kcal} / \mathrm{mole})$ is less than that of pantoprazole-BSA ( $-8.0 \mathrm{kcal} / \mathrm{mole})$ complex (Table 2$)$.

The effect of pantoprazole on nicotine and nicotine on pantoprazole binding to BSA are investigated in presence and absence of diazepam and warfarin sodium as one drug majorly binds to warfarin sodium site and the other one mainly to diazepam site. To summarize, pantoprazole is displaced by nicotine from its BSA binding pocket at a lesser extent than that of nicotine being displacement by pantoprazole. Secondly, the degree of displacement is higher in presence of the site specific probes diazepam and warfarin sodium. The evidence that pantoprazole displaces nicotine more from its binding pocket on BSA is a clear testimony of its higher affinity. These outcomes are strongly supported by computational evidences. In quantum chemical calculations, the dipole moment of pantoprazole is found to be higher than that of nicotine. As dipole moment amplifies the polar nature of a molecule and promotes hydrogen bond and non-bonded interactions in drug protein complexes [31], it is believed to increase the association constants of the former than that of the later with BSA in spectroscopic analysis. Small HOMO-LUMO gap and hence increased softness of pantoprazole also accounts for its stronger affinity for BSA than that of nicotine (Table 1) as a low gap marks low kinetic stability of the ligand and hence higher reactivity [32] [33]. Higher affinity of pantoprazole for BSA than that of nicotine in molecular docking is evident from Table 2 which corroborates the observation that nicotine displaces BSA bound pantoprazole less than nicotine being displaced by pantoprazole from its binding pocket in BSA.

\section{Conclusion}

This study focuses on the protein binding kinetics of nicotine and pantoprazole with bovine serum albumin to predict their interaction in vivo with human serum albumin. To determine the number of binding sites, association constants associated with each site, predominant binding site, displacement of one drug bound to bovine serum albumin by the other, in vitro equilibrium dialysis and UV spectroscopic techniques are employed. Computational tools further verify the results. Both of the test drugs are found to bind with either of the sites of the bovine serum albumin and predominantly bind to site-I and site-II. Moreover, the affinity of pantoprazole for site-I is proved to be higher than that of nicotine for the site-II. On the other hand, pantoprazole displaces nicotine from its binding pocket in bovine serum albumin at a higher extent than nicotine displacing pantoprazole from the two pockets. So, nicotine and pantoprazole compete for the different binding sites on bovine serum albumin and one increases the $\%$ free fraction of the other. As a result, concurrent administration of pantoprazole, while smoking might be beneficial since pantoprazole will help reduce gastric acidosis and GERD more effectively, is often caused by smoking.

\section{Authors' Note}

Kanij Nahar Deepa is the principle investigator of the manuscript, carries out 
primary literature review, and conceives the idea. Md. Abu Sufian did the computational analysis. Sabia Nawsheen carried out experiments, wrote the first draft of this manuscript. This experiments received no grant, or form of payment to conduct any analysis. All authors read and approved the manuscript. The datasets used and/or analyzed during the current study are available from the corresponding author on reasonable request.

\section{Acknowledgements}

The authors of this manuscript are grateful to the Red-Green Research Centre, Dhaka, Bangladesh for the laboratory facilities and IEERD of University of Asia Pacific for the support.

\section{Conflicts of Interest}

The authors declare no conflicts of interest regarding the publication of this paper.

\section{References}

[1] Holbrook, B.D. (2016) The Effects of Nicotine on Human Fetal Development. Birth Defects Research Part C: Embryo Today: Reviews, 108, 181-192.

https://doi.org/10.1002/bdrc.21128

[2] Grana, R., Benowitz, N. and Glantz, S.A. (2014) E-Cigarettes: A Scientific Review. Circulation, 129, 1972-1986.

[3] Mayer, B. (2014) How Much Nicotine Kills a Human-Tracing Back the Generally Accepted Lethal Dose to Dubious Self-Experiments in the Nineteenth Century. Archives of Toxicology, 88, 5-7. https://doi.org/10.1007/s00204-013-1127-0

[4] Caponnetto, P., Campagna, D., Papale, G., Russo, C. and Polosa, R. (2012) The Emerging Phenomenon of Electronic Cigarettes. Expert Review on Respiratory Medicine, 6, 63-74. https://doi.org/10.1586/ers.11.92

[5] Jerry, J.M., Collins, G.B. and Streem, D. (2015) E-Cigarettes: Safe to Recommend to Patients. Cleveland Clinic Journal of Medicine, 82, 521-526.

https://doi.org/10.3949/ccjm.82a.14054

[6] Schraufnagel, D.E., Blasi, F., Drummond, M.B., Lam, D.C., Latif, E., Rosen, M.J., et al. (2014) Electronic Cigarettes, a Position Statement of the Forum of International Respiratory Societies. American Journal of Respiratory and Critical Care Medicine, 190, 611-618.

[7] Cheer, S.M., Prakash, A., Faulds, D. and Lamb, H.M. (2003) Pantoprazole: An Update of Its Pharmacological Properties and Therapeutic Use in the Management of Acid-Related Disorders. Drugs, 63, 101-133. https://doi.org/10.2165/00003495-200363010-00006

[8] Kahrilas, P.J. and Gupta, R.R. (1990) Mechanisms of Acid Reflux Associated with Cigarette Smoking. Gut, 31, 4-10. https://doi.org/10.1136/gut.31.1.4

[9] Levitt, D.G. and Levitt, M.D. (2016) Human Serum Albumin Homeostasis: A New Look at the Roles of Synthesis, Catabolism, Renal and Gastrointestinal Excretion, and the Clinical Value of Serum Albumin Measurements. International Journal of General Medicine, 9, 229-255. https://doi.org/10.2147/IJGM.S102819

[10] Chavesa, O.A., Da-Silvaa, V.A., Sant'Annaa, C.M.R., Ferreiraa, A.B.B., Ribeiro, 
T.A.N., De-Carvalho, M.G., Cesarin-Sobrinho, D. and Netto-Ferreira, J.C. (2017) Binding Studies of Lophirone B with Bovine Serum Albumin (BSA): Combination of Spectroscopic and Molecular Docking Techniques. Journal of Molecular Structure, 1128, 606-611. https://doi.org/10.1016/j.molstruc.2016.09.036

[11] Shargel, L., Wu-pong, S. and Andrew, Y. (2012) Physiologic Drug Distribution and Protein Binding, Applied Biopharmaceutics and Pharmacokinetics. $6^{\text {th }}$ Edition, The Mcgraw Hill Companies, United States, Vol. 10, 205-248.

[12] Liu, B., Yang, C., Yan, X., Wang, J. and Ly, Y. (2012) Interaction of Avelox with Bovine Serum Albumin and Effect of the Coexistent Drugs on the Reaction. International Journal of Analytical Chemistry, 2012, Article ID 408057.

[13] Chen, C., Xiang, B., Yu, L., Wang, T. and Zhao, B. (2008) The Application of Two-Dimensional Fluorescence Correlation Spectroscopy on the Interaction between Bovine Serum Albumin and Paeonolum in the Presence of Fe (III). Spectroscopy, 41, 385-392.

[14] De-Vane, C.L. (2002) Clinical Significance of Drug Binding, Protein Binding, and Binding Displacement Drug Interactions. Psychopharmacology Bulletin, 36, 5-21.

[15] Trainor, G.L. (2007) The Importance of Plasma Protein Binding in Drug Discovery. Expert Opinion on Drug Discovery, 2, 51-64.

[16] Deepa, K.N., Hossain, M.K., Amran, M.S. and Kabir, S. (2014) In-Vitro Model for Studying Interactions between Ketorolac and Omeprazole with Bovine Serum Albumin by UV-Spectroscopic Method. Bangladesh Pharmaceutical Journal, 17, 92-98. https://doi.org/10.3329/bpj.v17i1.22323

[17] He, X.M. and Carter, D.C. (1992) Atomic Structure and Chemistry of Human Serum Albumin. Nature, 358, 209-215. https://doi.org/10.1038/358209a0

[18] Kudelski, A. (2003) Influence of Electrostatically Bound Proteins on the Structure of Linkage Monolayers: Adsorption of Bovine Serum Albumin on Silver and Gold Substrates Coated with Monolayers of 2-Mercaptoethanesulphonate. Vibrational Spectroscopy, 33, 197-204. https://doi.org/10.1016/j.vibspec.2003.09.003

[19] Sharma, Y.R. (2007) Ultraviolet and Visible Spectroscopy, Elementary Organic Spectroscopy. 16th Edition, S Chand and Company Limited, New Delhi, 9-59.

[20] Frisch, M.J., Trucks, G.W., Schlegel, H.B., Scuseria, G.E., Robb, M.A., Cheeseman, J.R., et al. (2009) D.J. Fox, Gaussian 09. Revision C.01, Gaussian Inc., Wallingford.

[21] Becke, A.D. (1988) Density-Functional Exchange-Energy Approximation with Correct Asymptotic Behavior. Physical Review A, 38, 3098-3100. https://doi.org/10.1103/PhysRevA.38.3098

[22] Becke, A.D. (1993) A New Mixing of Hartree-Fock and Local Density Functional Theories. The Journal of Chemical Physics, 98, 1372-1377.

https://doi.org/10.1063/1.464304

[23] Easton, R.E., Giesen, D.J., Welch, A., Cramer, C.J. and Truhlar, D.G. (1996) The MIDI Basis Set for Quantum Mechanical Calculation of Molecular Geometries and Partial Charges. Theoretical Chemistry Accounts, 93, 281-301. https://doi.org/10.1007/BF01127507

[24] Parr, R.G. and Weitao, Y. (1989) Density-Functional Theory of Atoms and Molecules. Oxford University Press, New York, 24-25.

[25] Pearson, R.G. (1995) The HSAB Principle-More Quantitative Aspects. Inorganica Chimica Acta, 240, 93-98.

[26] Guex, N. and Peitsch, M.C. (1997) Swiss Model and the Swiss-Pdb Viewer: An Environment for Comparative Protein Modeling. Electrophoresis, 18, 2714-2723. 
https://doi.org/10.1002/elps.1150181505

[27] Trott, O. and Olson, A. (2010) AutoDock Vina: Improving the Speed and Accuracy of Docking with a New Scoring Function, Efficient Optimization and Multithreading. Journal of Computational Chemistry, 31, 455-461.

[28] Wang, Y., Xiao, J., Suzek, T.O., Zhang, J., Wang, J., Bryant, S.H., Wang, Y., Xiao, J., Suzek, T.O., et al. (2009) PubChem: A Public Information System for Analyzing Bioactivities of Small Molecules. Nucleic Acids Research, 37, 623-633. https://doi.org/10.1093/nar/gkp456

[29] Pearson, R.G. (1986) Absolute Electronegativity and Hardness Correlated with Molecular Orbital Theory. Proceedings of the National Academy of Sciences, 83, 8440-8441. https://doi.org/10.1073/pnas.83.22.8440

[30] De-Lano, W.L. (2017) The PyMOL Molecular Graphics System. DeLanoSci., San Carlos.

[31] Biovia, D.S. (2015) Discovery Studio Modeling Environment. Dassault Syst. Release, San Diego, 4.

[32] Shawon, J., Khan, A.M., Rahman, A., Hoque, M.M., Khan, M.A.K., Sarwar, M.G., et al. (2016) Molecular Recognition of Azelaic Acid and Related Molecules with DNA Polymerase I Investigated by Molecular Modeling Calculations. Interdisciplinary Sciences, 10, 525-537.

[33] Aihara, J. (1999) Reduced HOMO-LUMO Gap as an Index of Kinetic Stability for Polycyclic Aromatic Hydrocarbons. The Journal of Physical Chemistry A, 103, 7487-7495. https://doi.org/10.1021/jp990092i 